\title{
Monitoring of Oxidation Steps of Ascorbic Acid Redox Reaction by Kinetics-Sensitive Voltcoulometry in Unsupported and Supported Aqueous Solutions and Real Samples
}

\author{
Jozef Orlický,* Katarína Gmucová,***† Ilja Thurzo,** and Juraj PavláSeK*** \\ *Institute of Molecular Physiology and Genetics, Slovak Academy of Sciences, Vlarska 5, 833 34, Bratislava, \\ Slovak Republic \\ **Institute of Physics, Slovak Academy of Sciences, Dúbravská cesta 9, 84511 Bratislava, Slovak Republic \\ ***Institute of Normal and Pathological Physiology, Slovak Academy of Sciences, \\ Vlárska 5, 83334 Bratislava, Slovak Republic
}

\begin{abstract}
Aqueous solutions of ascorbic acid in unsupported and supported aqueous solutions and real samples were studied by the kinetics-sensitive double-step voltcoulommetric method with the aim to contribute to a better understanding of its behavior in biological systems. The data obtained from measurements made on analytes prepared in the laboratory, as well as those made on real samples (some commercial orange drinks, flash of the fresh fruits) point to the redox reaction of $\mathrm{L}$-ascorbic acid $\left(\mathrm{L}-\mathrm{AH}_{2}\right)$ being very sensitive to both the presence of dissolved gaseous species $\left(\mathrm{O}_{2}, \mathrm{CO}_{2}\right)$ and the ionic strenght in the analyte. Either the dissolved gaseous species, or the higher ionic strength caused by both the presence of supporting electrolyte and increased total concentration of ascorbic acid, respectively, give birth to the degradation of L$\mathrm{AH}_{2}$. Naturally, the highest percentage of $\mathrm{L}-\mathrm{AH}_{2}$ was spotted in fresh fruit.
\end{abstract}

(Received September 11, 2002; Accepted December 9, 2002)

\section{Introduction}

Ascorbate, or vitamin $\mathrm{C}$, a well-known antioxidant is involved in the protection of the human organism against a variety of oxidative agents, especially free radicals. ${ }^{1,2}$ The oxidation of ascorbate usually takes place in a two-step reaction, in which electrons are transferred. The first step yields a relatively stable ascorbate free radical (AFR). ${ }^{3,4}$ In the second one, AFR donates a second electron, yielding dehydroascorbic acid (DHAA). ${ }^{5,6}$ These steps are reversible: two molecules of AFR can disproportionate, forming one DHAA and one ascorbate molecule. ${ }^{7}$ Over the past 20 years a large number laboratories demonstrated that voltammetric records of ascorbic acid (AA) could be carried out successfully by using chemically modified electrodes. Despite of that fact the kinetics of electron transfer are still quite sluggish and fast degradation of the attached or adsorbed materials results in a difficulty to be used for these purposes. Recently, the quantification of AA and total AA in food, fruits and spinach by capillary-zone electrophoresis and/or chromatography $\mathrm{y}^{8,9}$ has been reported.

The aim of the presented paper is to discuss the ascorbic acid redox reactions, which take place at the carbon fiber/ascorbic acid interface. The used experimental equipment combined two methods: stripping kinetics-sensitive double-step voltcoulometry (i.e. the transient charge response to the excitation pulse is sampled) and steady-state current voltammetry (i.e. the steady-state current is measured during the absence of an excitation pulse). During the measurement, the

† To whom correspondence should be addressed.

E-mail: Katarina.Gmucova@savba.sk transient charge $Q(t)$ related signal is sampled with the aim to obtain the desired response in the form

$$
\Delta q(E)=Q\left(t_{1}\right)-2 Q\left(5 t_{1}\right)+Q\left(9 t_{1}\right),
$$

where $t_{1}, 5 t_{1}, 9 t_{1}$ are the sampling times with respect to the trailing edge of the excitation pulse and $E$ is the potential of the working electrode. Each measuring period is preceded by a single-shot measurement of the steady-state current $(I)$ with the excitation pulse being switched off. By that way, the experimental equipment enables us to obtain the following two data sheets from a single scan of the potential: the correlated differential charge $(\Delta q(E))$ and the steady-state voltammetric wave $(I(E)){ }^{10,11}$ These data, detached to the same period from the sample preparation provide valuable information about the kinetics of the redox reaction under consideration ${ }^{12}$ as well as about the percentage of individual species dissolved in the measured sample.

\section{Experimental}

Reagents

The water used to prepare of solutions was purified by an Elix-S-system (Millipore), or was purchased from commercial sources as aqua pro injectione (API-Hoechst, Biotika Slov. Lupca). The chemicals used in the presented study were of the highest commercially available purity: L-ascorbic acid (AA) and sodium chloride (suprapure) from Merck (Darmstadt, Germany), dopamine (DA, 3-hydroxytyramin $\cdot \mathrm{HCl}$ p.a.) from SERVA, Feinbiochemica Heidelberg and manitol (D-MAN, MW 212.2) synthesized by J. Kubala, Bratislava, Slovak 
Republic.

Care was taken to prevent possible contamination by carbon dioxide. The solutions were bubbled ( $21 / \mathrm{min})$ with argon at room temperature for $10-12$ min either in the stock one and/or in the electrode-cell compartment.

\section{Microelectrodes}

All of the experiments were performed in a two-electrode cell compartment with a carbon fiber microelectrode as a working electrode and an $\mathrm{Ag} / \mathrm{AgCl}$ wire as a standard reference electrode. The working electrode was formed of a glass micropipette with up to eight carbon fibers (diameter of each fiber being approximately $7-8 \mu \mathrm{m}$ ). The pipette tip (diameter $100-150 \mu \mathrm{m}$ ) was filled with paraffin wax to prevent liquid from seeping inside around the carbon fibers. The exposed surfaces of the filaments were treated electrochemically before starting the experiments. First, a cathodic potential of $-0.8 \mathrm{~V}$ was applied for $20 \mathrm{~s}$, followed by a triangular waveform of 0 to $+3 \mathrm{~V}$ for $5 \mathrm{~s}$; finally, an anodic potential of $+1.5 \mathrm{~V}$ was applied for $5 \mathrm{~s}$.

\section{Voltammetric instrumentation}

In order to ease the reader's insight into what will be presented further, we summarize the basic principles of the double-step voltcoulommetry (DSVCM) from our previous works. ${ }^{10-12}$ It is common to assume that the transient response of an electrochemical system consists of the following current contributions: the diffusion current, the capacitive current, and the steady-state current. The problem to be overcome in all voltcoulometric experiments is the necessity to suppress the parasitic signal due to charging the electrical double-layer near the electrode, i.e. the contribution of the capacitive current. One of the essential features of DSVCM is the abillity to perform that suppression. Despite the fact that in the DSVCM method that problem is solved by the filtering scheme that emphasizes the diffusion current, an appropriate choice of the parameter settings is mandatory to improve the figure of merit of the DSVCM method. Since choosing slower sampling modes is highly recommended, the reported results correspond to the measurements made for sampling time $t_{1}=15.96 \mathrm{~ms}$. All of the experimental data correspond to the potential-step amplitude $\Delta E$ $=-0.085 \mathrm{~V}$; the quiescent potential $E$ was scanned at a rate of $0.0033 \mathrm{~V} / \mathrm{s}$.

The main advantage of the DSVCM method is a high sensitivity to the kinetics of the redox reaction. To emphasize that fact, we produce as evidence the measurement of an aqueous solution of ascorbic acid ( $1 \mathrm{mM} / \mathrm{l}$, as prepared-without deaireating by argon), by the following methods:

a) Cyclic voltammetry

b) DSVCM method

c) Steady-state voltammetric wave

d) Derivative steady-state voltammetry.

The experimental data, which were obtained on the same analyte, in the same electrochemical cell equipped by the same electrode system, are shown in Fig. 1. The distinct signals observed within the potential range from $-1 \mathrm{~V}$ to $+0.6 \mathrm{~V}$, proceeding from a negative potential to a positive one, can be ascribed to the redox reactions of the following species: $\mathrm{H}^{+}$, $\mathrm{L}-\mathrm{AH}_{2}$, and DHAA. It is evident, that while the steady state voltammetric wave, as well as the derivated voltammetric signal (derived mathematically from the voltammetric wave), yield only unexpressive information about the redox reaction of $\mathrm{L}-\mathrm{AH}_{2}$, the DSVCM is able to detect all of the three redox reactions taking place in the prepared analyte. The detection limits of the methods used in our experiment differ from each

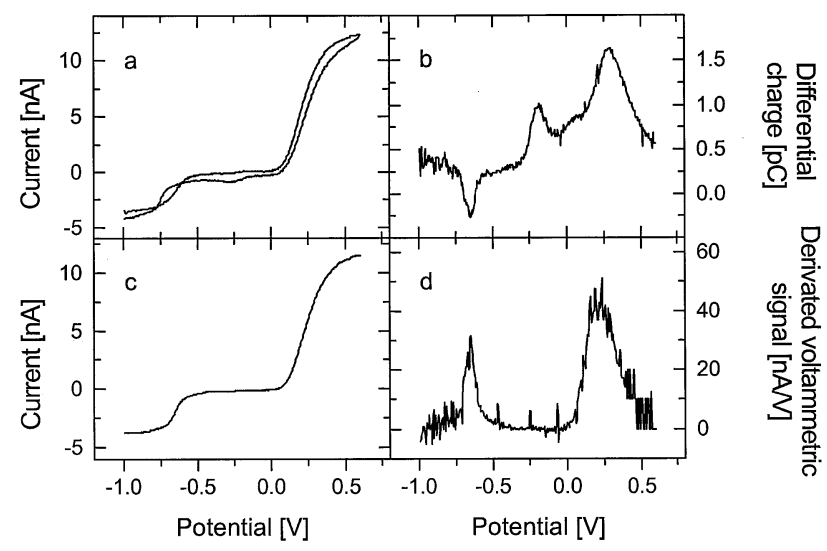

Fig. 1 Electrochemical measurements of AA $(1 \mathrm{mM} / \mathrm{l})$. a, Cyclic voltammogram; scan rate $=0.1 \mathrm{~V} / \mathrm{s} ; \mathrm{b}$, DSVCM signal; $\mathrm{c}$, steadystate voltammetric wave; scan rate $=0.0033 \mathrm{~V} / \mathrm{s} ; \mathrm{d}$, derivative voltammetry signal.

other as a consequence of the fact that the time dependance of the transient charge, registered after the sampling pulse being applied to the reference electrode, does not obey the behavior theoretically predicted by the Cottrell equation. The differences in the measured signal are sufficiently expressive. Moreover, if the transient response behaves superlineary with time, we can observe a negative peak - e.g. for the redox reaction of $\mathrm{H}^{+}$in an analyte prepared withought deaireating by argon (Fig. 1). On the other hand, for the standard redox reaction (sublinear transient response), positive peaks are observed. The kineticsdiscrimating feature of DSVCM was detailed in our previous works. ${ }^{10-12}$ Here, it is worth mentioning that the simultaneous application of at least two electrochemical methods, one of them sensitive to kinetics, can be useful for a better understanding of the redox processes taking place in the analyte under consideration.

Finally, a comparison of the DSVCM measurement with the standard cyclic voltammogram, plotted for the potential scanned from $-1 \mathrm{~V}$ to $+0.6 \mathrm{~V}$ and back to $-1 \mathrm{~V}$ with the scan rate being equal to $0.1 \mathrm{~V} / \mathrm{s}$, is given in Fig. 1.

\section{Results and Discussion}

DSVCM behavior of L-ascorbic acid on a carbon fiber microelectrode in unsupported aqueous solutions

In agreement with our previous work, ${ }^{10,11}$ the results obtained by different techniques suggested that the voltammetric (voltcoulometric) signal present at the negative potential $(\sim-0.2$ $\mathrm{V}$ ) corresponds to the oxidation of the $\mathrm{L}-\mathrm{AH}_{2}$ species reduced during a negative pulse, while the signal present at the positive potential $(\sim+0.25 \mathrm{~V})$ was estimated as being the apparent redox potential of DHAA. In an effort to highlight the DSVCM abilities in the detection of the redox reactions, several experiments on a deaireated aqueous solution of AA were conducted.

Though the fundamental behavior of AA on the carbon fiber microelectrode was reported in our previous work, ${ }^{10,11}$ details of the behavior, especially with respect to the dose-response of AA concentrations, remained unresolved. DSVCM voltcoulommograms for eight different concentrations of AA are plotted in Fig. 2. In the presence of an excess amount of AA in the range $0.5-2 \mathrm{mM} / 1$ (Fig. 2, curves a-d) similar portions 


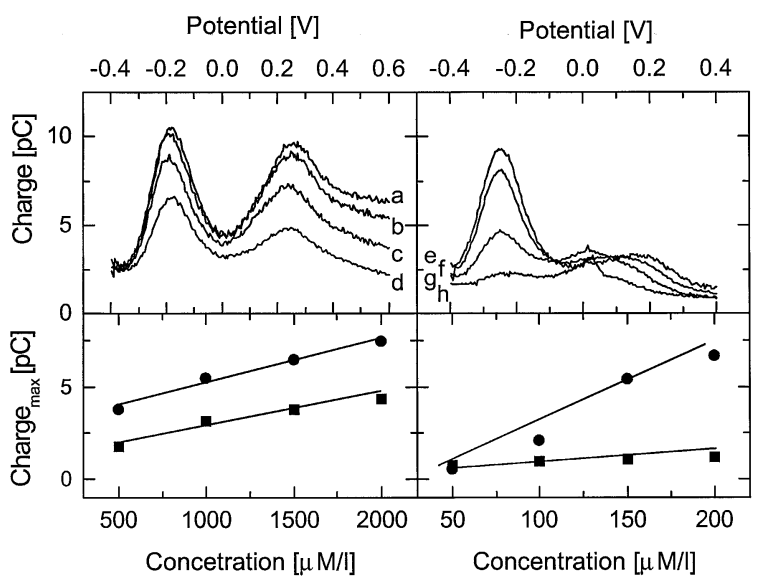

Fig. 2 DSVCM measurements of AA within the concentration ranges from $500 \mu \mathrm{M} / 1$ to $2000 \mu \mathrm{M} / 1$ and from $50 \mu \mathrm{M} / 1$ to $200 \mu \mathrm{M} / 1$, respectively, performed with two different electrodes (a, $2000 \mu \mathrm{M} / 1$; b, $1500 \mu \mathrm{M} / \mathrm{l} ; \mathrm{c}, 1000 \mu \mathrm{M} / \mathrm{l} ; \mathrm{d}, 500 \mu \mathrm{M} / \mathrm{l}$; e, $200 \mu \mathrm{M} / \mathrm{l}$; f, $150 \mu \mathrm{M} / \mathrm{l}$; g, $100 \mu \mathrm{M} / \mathrm{l} ; \mathrm{h}, 50 \mu \mathrm{M} / \mathrm{l})$. In the lower part of the figure, respective calibration curves for DHAA $(\bullet)$ and $\mathrm{L}-\mathrm{AH}_{2}(\bullet)$ are plotted.

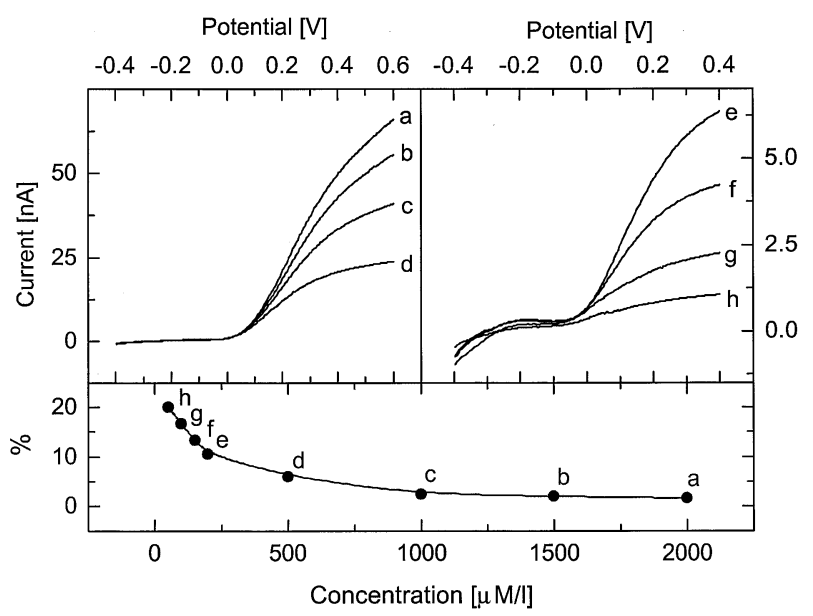

Fig. 3 Steady-state voltammetric waves corresponding to DSVCM measurements on AA (curves $a-h$ ) from Fig. 2. In the lower part of figure the percentage of $\mathrm{L}-\mathrm{AH}_{2}$ referred to the total ascorbic acid is shown.

of the voltcoulometric signals were observed at both the negative potential $(\sim-0.20 \mathrm{~V}$, correspondig to redox reaction of $\left.\mathrm{L}-\mathrm{AH}_{2}\right)$ and the positive one $(\sim+0.25 \mathrm{~V}$, corresponding to redox reaction of DHAA), respectively. Experiments performed under similar conditions, except for the presence of micromolar concentrations of AA (Fig. 2, curves e-h), provide a significant voltammetric response mainly within the negative potentials $(\sim-0.25 \mathrm{~V})$, i.e. from the redox reaction of $\mathrm{L}-\mathrm{AH}_{2}$. Within the concentration range of $0.05-0.2 \mathrm{mM} / \mathrm{l}$, the slope of the analytical curve corresponds to about $43 \mathrm{pC} / \mathrm{mM}$, and the linear correlation coefficient is 0.990 . On the contrary, the linearity of the peak amplitude and the slope at positive potentials in this concentration range are lower: the slope of the analytical curve is $2.7 \mathrm{pC} / \mathrm{mM}$ and the linear correlation coefficient is 0.977 . In the concentration range from 0.5 to $2 \mathrm{mM} / \mathrm{l}$, the behavior of the peak at positive potential is enhanced with respect to the sensitivity and linearity. The slopes of the analytical curves for either the DHAA or $\mathrm{L}^{-\mathrm{AH}_{2}}$ peaks correspond to 2.4 and 1.7

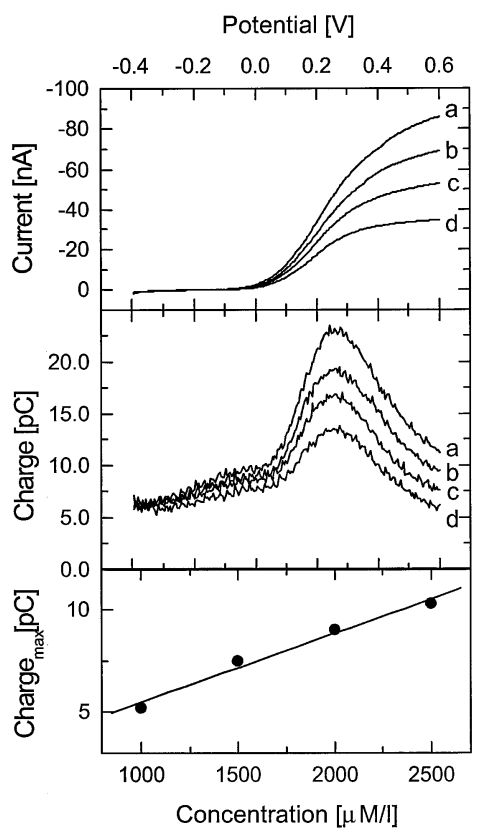

Fig. 4 Steady-state voltammetric waves, DSVCM signals and respective calibration curve for supported $(150 \mathrm{mM} / 1 \mathrm{NaCl}) \mathrm{AA}$ solutions of the following concentrations: a, $2.5 \mathrm{mM} / \mathrm{l} ; \mathrm{b}, 2.0 \mathrm{mM} / \mathrm{l}$; c, $1.5 \mathrm{mM} / 1$ and d, $1.0 \mathrm{mM} / 1$.

$\mathrm{pC} / \mathrm{mM}$, and the linear correlation coefficients are 0.984 and 0.978 , respectively.

The steady-state voltammetric waves, correspoding to the above-mentioned concentrations of total AA, are plotted in the upper part of Fig. 3. From the half-wave potentials of the individual components (DHAA, $\mathrm{L}-\mathrm{AH}_{2}$ ) of the spectra, the percentage of $\mathrm{L}-\mathrm{AH}_{2}$ was evaluated as referring to the total AA for various concentrations of AA in solution. The resulting curve is plotted in the lower part of Fig. 3. All of the experimental data, obtained for two different working electrodes, seem to indicate that the percentage of $\mathrm{L}-\mathrm{AH}_{2}$ can be approximated by a curve rising toward to the lower concentrations of the total AA. The obtained data are in good agreement with published values. ${ }^{13}$

In one of our previous studies ${ }^{11}$ we suggested introducing the ratio of the maximum of the DSVCM peak height to the limiting steady-state current multiplied by the sampling time, $R_{\beta}$ $=\Delta Q_{\max } / I_{\mathrm{lim}} t_{1}$. This parameter seems to be an effective tool for estimating both the deviations of the measured transient charge from the ideal behavior (fulfilling the Cottrell equation) and the propriety of using the DSVCM method under the given conditions. Generally, it can be postulated that the higher is the parameter $R_{\beta}$ in the absolute value, the higher is the sensitivity of DSVCM method, compared to ordinary steady-state voltammetry. From both the Figs. 2 and 3 the following values of parameter $R_{\beta}$ were obtained: 0.6 for $\mathrm{L}_{-} \mathrm{AH}_{2}, 0.004$ for DHAA.

DSVCM response of the L-ascorbic acid in the presence of $\mathrm{NaCl}$ as a supporting electrolyte

DSVCM protocols for ascorbate ions in a $150 \mathrm{mM} / 1 \mathrm{NaCl}$ solution were obtained in the concentration range from 1.0 to $2.5 \mathrm{mM} / 1$ under the same experimental conditions, as described in the preceding section. The resulting voltcoulometric response is expressive only within the positive potential $(\sim+0.30$ V) (see Fig. 4). The corresponding steady-state voltammetric waves are plotted in the upper part of Fig. 4, from which we can 


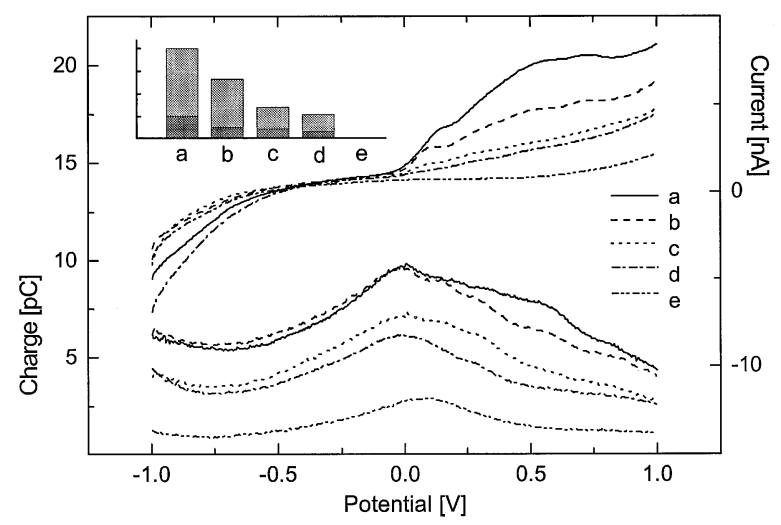

Fig. 5 Plot of the DSVCM signal as well as the voltammetric wave obtained from measurements made on a juice obtained from a fresh orange fruit (a) and some commercial orange juices (b, $100 \%$ orange juice; c, $10 \%$ orange juice with artificially added ascorbic acid; d, $13 \%$ orange juice; e, drink with orange flavor). Normalized contents of both the DHAA (light gray column) and $\mathrm{L}-\mathrm{AH}_{2}$ (gray column) for respective samples a, b, c, d and e as the inset.

deduce, that the percentage of $\mathrm{L}_{-} \mathrm{AH}_{2}$, as refered to the total AA, is negligible. The obtained results indicate that $\mathrm{NaCl}$ as a supporting electrolyte is really not an appropriate medium when dealing with AA determinations for concentrations lower than $0.2 \mathrm{mM} / \mathrm{l}$. An explanation of such behavior may reside in the fact that the electrical double-layer, formed on the surface of the working electrode, is altered owing to specific ion adsorption. Consequently, the sensitivity of the DSVCM method is lower compared to the unsupported conditions.

\section{Real samples measurements}

The proposed method was applied to beverages of a commercial fruit juice, and/or a fresh fruit with the aim to determine the percentage of AA substrates immediately, as well as after the aging by storage in air. The DSVCM protocols for some orange juices, obtained for identical conditions while immersing the same electrode into the juice-sample, are plotted in Fig. 5. In the lower part, the correlated differential charge, and in the upper part the steady-state voltammetric waves are shown. Ex hypothesi, if the amount of the total AA in the sample decreases, the voltammetric signal is decreasing. The best-resolved signal was recorded under the circumstances when the juice was obtained from fresh orange fruit immediately before measurements in our laboratory; it is depicted as a. The other orange juices, bought in a supermarket, were marked as follows: b, $100 \%$ orange juice; c, $10 \%$ orange juice with artificially added ascorbic acid; $d, 13 \%$ orange juice; e, drink with orange flavor. From the half-wave potential, the normalized contents of both the DHAA (light gray column) and $\mathrm{L}-\mathrm{AH}_{2}$ (gray column) were calculated for respective samples a, b, c, d and e, and plotted as the inset of Fig. 5. The selectivity in the DSVCM signal is low, probably due to microelectrode fouling by unspecified ingredients present in commercial drinks.

Finally, orange and kiwi fruit were measured by the following procedure. Just before the measurement, fresh fruit was scraped on a small area $\left(\mathrm{a}\right.$ few $\mathrm{mm}^{2}$ ). Then, electrochemically treated electrodes were stuck into the flesh of the fruit and the measurement was started as soon as possible. The obtained signals are plotted in Fig. 6 by full lines. When the measurement was stopped, both the working and reference electrodes, mounted on a common holder, were taken out from the fruit to avoid any excessive pollution of the working

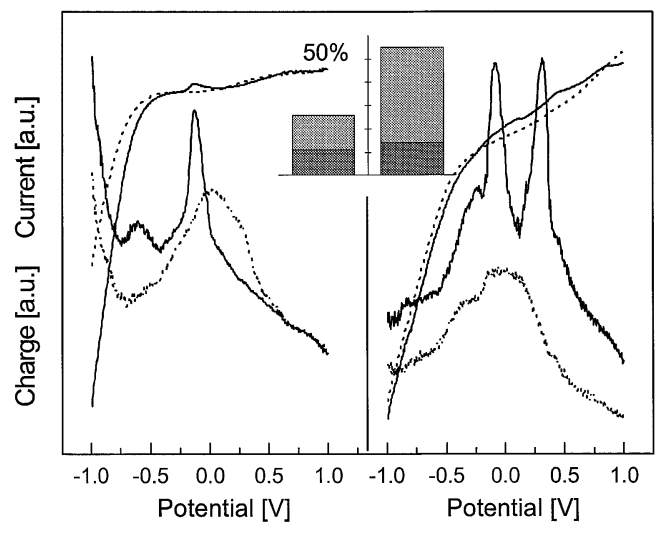

Fig. 6 Aging of ascorbic acid in both the orange and kiwi fruit. The full lines represent measurements (both the DSVCM signal and voltammetric wave, respectively) on the fresh fruit; the dotted lines were obtained after half an hour exposition of the scraped fruit to the air and light. The percentage of $\mathrm{L}_{-} \mathrm{AH}_{2}$ (referring to the total AA) in the fresh fruit (light gray column) and after half an hour exposition of the scraped fruit into the air and light (gray column) in orange and kiwi fruit as the inset.

electrode surface by some adsorbed species. After half an hour exposition of the scrapped fruit at room temperature into the air, the same electrode system was used to obtain data, plotted in Fig. 6 by the dotted line. From the half-wave potential of the steady-state voltammetric wave, the percentage of $\mathrm{L}_{-} \mathrm{AH}_{2}$ referred to the total AA was calculated. The results are depicted as the inset of Fig. 6. A fast degradation of DHAA was observed in both the orange and kiwi fruits exposed for half an hour to the air; its percentage can be estimated as being approximately $10-12 \%$ of the total AA, irrespective of the primary percentage in the fresh fruit. These results correspond to published values. ${ }^{14,15}$ Because the reference $\mathrm{Ag} / \mathrm{AgCl}$ electrode used in all our experiments was not saturated, the potentials of the maximum charge for the unsupported and supported aqueous solutions, as well as for the real samples may differ from each other. Also, the presence of background in the obtained spectra may cause a visible shift of the observed potential of the maximum charge.

\section{DSVCM behavior of AA, DA and D-MAN in the unsupported mixture}

For the sake of understanding the various aspects that can affect the determination of dissolved species by the DSVCM technique, we investigated the effects which take place in a deoxygenated mixture solution, composed of $1 \mathrm{mM} / 1 \mathrm{AA}$, $20 \mu \mathrm{M} / 1 \mathrm{DA}$ and $2.5 \mathrm{mM} / 1$ 1-nitro-D-manitol. The obtained voltcoulommograms have distinct resolved peaks at negative potentials corresponding to D-MAN, $\mathrm{H}^{+}$, and $\mathrm{L}-\mathrm{AH}_{2}$ redox reactions, as well as a couple of overlapping peaks at positive potentials, matching the signals coming from both the DHAA and DA redox reactions, respectively (Fig. 7).

The DSVCM method was applied to the above-mentioned mixture in both the oxidation (full line) and reduction (dotted line) regimes. Such a couple of DSVCM spectra, obtained for the potential step amplitude of $85 \mathrm{mV}$ (oxidation) and $-85 \mathrm{mV}$ (reduction), respectively, provide information about the reversibility of the redox reaction under consideration. ${ }^{10}$ All of the redox reactions, belonging to the above-mentioned mixture, are quasi-reversible. The confrontation of the voltcoulometric charge signal and the derivative current signal suggests the possibility that the individual redox reactions may differ in 


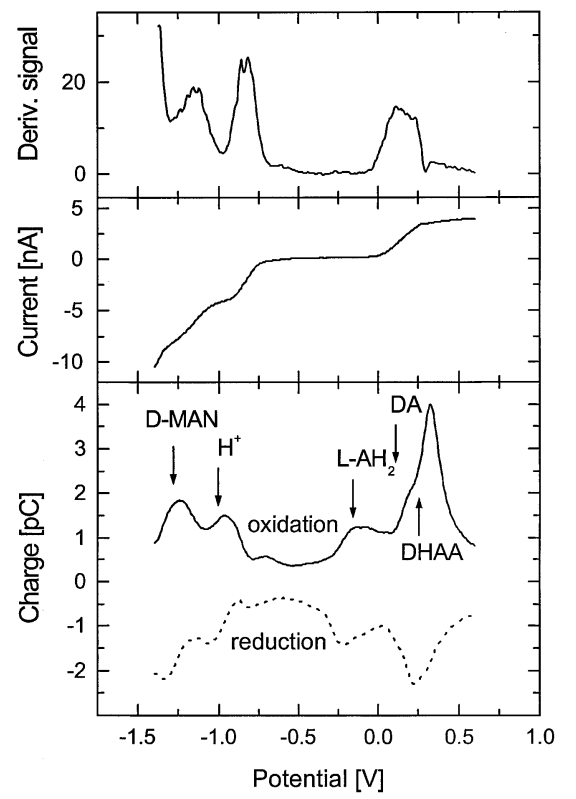

Fig. 7 Voltcoulograms (lower part) along with the respective steady-state voltammetric wave (middle part) and the corresponding steady-state derivative voltammetry signal (upper part) recorded for a mixture of manitol $(2.5 \mathrm{mM} / \mathrm{l})$, ascorbic acid $(1 \mathrm{mM} / \mathrm{l})$ and dopamine $(20 \mu \mathrm{M} / \mathrm{l})$.

respective kinetics. ${ }^{10-12}$

\section{Conclusions}

The findings mediated by the DSVCM method in the unsupported, supported and real samples provide the following conclusions:

(1) Under the optimal experimental conditions (the solutions deaireated by pure argon) the DSVCM produced better recording properties of the unmodified carbon fiber microelectrode in solutions either with or without a supporting electrolyte, probably due to the relatively higher resistance of the analyte after removing the dissolved carbon dioxide.

(2) A linear relationship between the AA concentrations and the peak heights in a relatively wide concentration range was found.

(3) The detection limits of total AA for both the $\mathrm{L}^{-\mathrm{AH}_{2}}$ and DHAA are at the rank of tens $\mu \mathrm{M} / \mathrm{l}$.

(4) The sensitivity of the DSVCM method to the DHAA is lower than the sensitivity of ordinary steady-state voltammetry. On the other hand, the sensitivity to $\mathrm{L}-\mathrm{AH}_{2}$ is higher for DSVCM method. This divergence is caused by different kinetics of the respective redox reaction.
(5) The measurments were also performed on real samples e.g. some commercial orange drinks, the juice obtained from a fresh fruit in our labolatory and directly inside the fresh fruits, with both the working and reference electrodes immersed in them. The data obtained support the concept of the redox reaction of $\mathrm{L}-\mathrm{AH}_{2}$ responsible for the peak at negative potential as being very sensitive to the presence of $\mathrm{O}_{2}, \mathrm{CO}_{2}$ and to the ionic strength in the analyte. This peak was predominantly detected under in situ conditions in fresh fruits.

(6) The detection of AA by the DSVCM in foodstuffs, beverages and pharmaceuticals can serve as a quality index, varying during the production period and the storage stage.

(7) The DSVCM method seems to be useful for either basic research and/or practical use.

The applicability of this method to the study of the charge transfer, the ascorbate radicals, the neurotransmitters and other electroactive substances in vitro as well as in vivo is a challenge for further improvements of the technique.

\section{Acknowledgements}

The authors wish to thank grant agency VEGA for the financial support of projects Nos. 2/1013/22, 2/6054/99, 2/1011/22.

\section{References}

1. H. Padh, Biochem. Cell. Biol., 1990, 68, 1166.

2. R. C. Rose and A. M. Bonde, FASEB J., 1993, 7, 1135.

3. B. H. J. Bielski, "Ascorbic Acid: Chemistry, Metabolism and Uses", 1982, American Chem. Soc., Washington, D.C.

4. G. R. Buettner, Arch. Biochem. Biophys., 1993, 300, 535.

5. M. Levine, N. Engl. J. Med., 1986, 314, 892.

6. J. C. Deutsch, Anal. Biochem., 1998, 260, 223.

7. C. P. Andrieux and J. M. Saveant, J. Electroanal. Chem., 1978, 93, 160.

8. P. A. Marshall, V. C. Trenerry, and C. O. Thompson, J. Chromatogr. Sci., 1995, 33, 426.

9. T. Liao, Ch. M. Jiang, M. Ch. Wu, J. Y. Hwang, and H. M. Chang, Electrophoresis, 2001, 22, 1484.

10. I. Thurzo, K. Gmucová, J. Orlický, and J. Pavlásek, Rev. Sci. Instr., 1999, 70, 3723.

11. I. Thurzo, K. Gmucová, J. Orlický, and J. Pavlásek, J. Electroanal. Chem., 2001, 514, 26.

12. K. Gmucová, I. Thurzo, J. Orlický, and J. Pavlásek, Electroanaysis, 2002, 14, 943.

13. H. M. Sorouraddin, A. Hibara, M. A. Proskurnin, and T. Kitamori, Anal. Sci., 2000, 16, 1033.

14. M. Bode, L. Cunningham, and R. C. Rose, Clin. Chem., 1990, 36, 1807.

15. M. M. Van Duijn, J. Van der Zee, J. VanSteveninck, and Van den Broek, J. Biol. Chem., 1998, 273, 13415. 